\title{
Influence of the Aggregate-Pouring Sequence on the Efficiency of Plugging Inundated Tunnels through Drilling Ground Boreholes
}

\author{
Xiangming Jiang ${ }^{1}$, Shuang Hui ${ }^{2}$, Wanghua Sui ${ }^{1, * \mathbb{D}}$, Zhiyuan Shi ${ }^{1,3}$ and Jiahao Wang ${ }^{4}$ \\ 1 School of Resources and Geosciences, Institute of Mine Water Hazards Prevention and Controlling \\ Technology, China University of Mining and Technology, Xuzhou 221116, China; jxm2007613@163.com (X.J.); \\ sizhiyuan@cctegxian.com (Z.S.) \\ 2 Environmental Engineering Company, China Design Group Co., Ltd., Nanjing 210001, China; \\ binghuyise@126.com \\ 3 Department of Hydrogeology, Xi'an Research Institute, CCTEG, Xi'an 710000, China \\ 4 Department of Municipal Engineering Design, Tongji Architectural Design (Group) Co., Ltd., \\ Shanghai 200092,China; wjh_phoenix@163.com \\ * Correspondence: suiwanghua@cumt.edu.cn; Tel.: +86-139-5219-9519
}

Received: 28 July 2020; Accepted: 25 September 2020; Published: 27 September 2020

\begin{abstract}
This paper presents an experimental and field investigation on the efficiency of plugging by pouring aggregate in different sequences through multiple boreholes in a tunnel with flowing water. There have been controversies surrounding the selection of the pouring order for different particle sizes of aggregates and the order in different boreholes. A visualized experimental setup is used to investigate the influence of the pouring orders on the efficiency of plugging through multiple boreholes under the flowing-water condition. A case study of the salvage of a flooded mine using ground directional boreholes was investigated and compared with the experimental results. The water-pressure difference at the aggregate-capping moment, when fine aggregate was poured first and coarse aggregate later, was relatively small, compared to that when fine aggregate was poured upstream and coarse aggregate, downstream. The result implies that the efficiency of plugging with the order of pouring fine aggregate first and coarse aggregate later in different boreholes is better than that with the order of pouring fine aggregate upstream and coarse aggregate downstream. When the poured aggregate is about to be capped, increasing the pouring intensity with the same or a larger particle size is more conducive to capping. The case study shows that pouring fine materials in the early stage reduced the cross-sectional area; in the later stage, the aggregate particle size was gradually increased, which can be helpful in forming an effective water-barrier section in the tunnel. The pouring of aggregate provided a base for cement grouting to form a water-plug section with a length of $106 \mathrm{~m}$, resulting in a sealing efficiency of $100 \%$ for the case.
\end{abstract}

Keywords: groundwater inrush; inundated tunnel; efficiency of plugging; pouring aggregate; order of pouring; water pressure

\section{Introduction}

A disastrous, sudden inrush of groundwater often floods tunnels and even whole coal mines in their production and construction. Plugging and grouting can quickly and effectively control water hazards, separating the water-flooded area and production area. If the location of the inrush pathway is known, the plugging and grouting can be carried out in that pathway. However, the water-conducting pathway is difficult to discover in most situations. In these cases, emergency relief is usually implemented in a centralized roadway flooded with water. A centralized roadway here generally refers to a tunnel that 
concentrates the water inrush flow, where there are no other tunnels within $30 \mathrm{~m}$. The grouting and blocking of water are generally carried out in the underground channel under the hazardous condition of water flowing at high pressure and speed.

A grouting and sealing project in an inundated mine tunnel is usually implemented in two stages: pouring aggregate and grouting reinforcement. The purpose of pouring aggregate is to form a water-blocking section in the tunnel through the accumulation of aggregates and to change the pipeline flow to a permeate flow. The purpose of the grouting reinforcement is to block the voids in the accumulating mass to seal off the water, with cement slurry. Therefore, aggregate pouring is a necessary prerequisite for effective grouting, while effective grouting plays a key role in successful water blocking. Moreover, the selection of aggregate is a key issue in grouting-shutoff engineering. In a pouring project, both fine aggregates, such as fly ash and sand, and coarse aggregates, such as gravel and pebbles of various particle sizes, are generally used. In practice, the maximum particle size of the aggregate should generally not exceed $1 / 3$ to $1 / 4$ of the borehole's inner diameter [1,2]. The aggregate is usually inserted with a special orifice injection device and then carried by a moderate and stable water flow through the borehole. A water-solid mass ratio between 5:1 and 15:1 is often selected [1,2]. According to the groundwater-inrush flow rate and the roadway environment, different pouring sequences are often adopted, such as pouring coarse particles first and then pouring fine ones or vice versa, and pouring coarse particles upstream and fine particles downstream or vice versa. The ultimate aim is to achieve the best efficiency of plugging.

Case histories and experiences of controlling groundwater inrush using this approach in mine tunnels have been discussed by many researchers and engineers in the literature. The process of aggregate accumulation and plugging is divided into three stages: the bottom-laying stage, filling stage and plugging stage [3]. After the aggregate pouring is completed, the pipeline flow in the tunnel becomes a permeate flow $[1,2,4]$. Some new technologies and materials have been applied in the construction of the water-retention section in the inundated tunnel. The feasibility of using fine aggregate in the water-blocking treatment of a tunnel with flow water has been discussed [5]. Grouting from the directional drilling of boreholes on the ground for the rapid construction of a water-retention structure in the tunnel was used in a groundwater-inrush control project in different coal mines, such as in the Luotuoshan coal mine, Inner Mongolia; the Yuchang coal mine, Shaanxi; and the Panji coal mine No.2, Anhui [6-9]. The method of placing grouting bags at fixed points and inject quick-setting and high-strength grouts was used to achieve the rapid and controllable blocking of tunnels with high flow rates [10].

Research on the transportation and plugging mechanism of the solid-liquid mixture during the pouring of aggregates in a flooded tunnel requires knowledge of the hydraulics, sediment kinematics, solid-liquid two-phase flow and suspension rheology. The results will provide a theoretical basis for the control of tunnel-water inrush disasters and improving engineering design. Experimental investigations and numerical simulations have been widely used for understanding the process of pouring aggregate in a tunnel. Aggregate-pouring techniques, the mechanism of the grouting reinforcement of the accumulating aggregate mass and the criteria for judging the water-retention capacity of the water-blocking section have been investigated experimentally and theoretically [8]. Experiments of pouring aggregate into a horizontal pipeline through a single borehole showed that the factors influencing the efficiency of plugging, in descending order, were the aggregate particle size, followed by the initial velocity of the water flow and then the water-solid mass ratio [11]. An experimental setup for placing grouting bags has recently been developed [12]. The study of material transportation in horizontal pipes has shown some similarity with the movement and sedimentation of aggregates in tunnels. The Eulerian-Lagrangian method and turbulent liquid-solid slurries in horizontal pipes were used to investigate single-dispersed fine-particle flow at high concentrations [13-16]. A new two-fluid model (TFM) was proposed and later improved by Messa et al. [17] and Messa and Matoušek [18]. Their results confirmed that the TFM is an effective tool for engineering design by comparing with experimental results [18]. 
In order to better understand the migration of slurry in multi-void pores, many experimental studies have been carried out. For example, Mohtar et al. [19] and Jaffal et al. [20] pointed out that the non-uniformity of soil would create a preferred flow path for the spread of grout, leading to non-uniform grout coverage and thus affecting the effects of penetration grouting. Civan [21] studied various forces, velocities and other related factors that affect the appearance of pore media through experiments with two modes of flow of particles in and out of pores. Mohtar et al. [22] studied the post-grouting stability of bentonite in porous media and established the relationship between the yield stress of the bentonite suspension and the critical water conductivity. Bedrikovetskya et al. [23] and Lenchenkov et al. [24] studied the flow of colloids in water and porous media, and confirmed the validity of their proposed model, which can also be used to predict potential well plugging. Civan and Vafai [25] examined the characteristic features of the fundamental flow mechanisms and discussed the essential parameters of relevant modeling approaches for the transportation of gas through dense, porous media. These studies are helpful for understanding the deposition and transportation of aggregates in a tunnel with flowing water and why the order of adding grains sizes is important in plugging the channel.

The hydraulic-pipeline transport of slurries has certain similarities with the accumulation of aggregate in a tunnel with flowing water. The states of solid-liquid mixtures have been classified into three categories-homogeneous, intermediate and heterogeneous-based on the suspension flow of sand and gravel in the water [26]. Wasp et al. categorized the flow regimes of solid materials in pipelines into two types: homogeneous and heterogeneous [27]. Solid particles are divided into bed load or suspended load by Fei [28]. The transport of sand-water slurries along a horizontal pipeline has been investigated by many researchers, such as Soepyan et al. [29] and Zouaoui et al. [30]. The transition of slurry flow from heterogeneous to homogeneous was investigated by Miedema [31]. This literature on slurry flow in pipelines is helpful for understanding the movement and deposition of aggregates in a tunnel with flowing water.

The water-pressure changes along a pipeline can help in understanding the mechanism in the two-phase flow to illustrate friction loss, and the reduction of pressure in a turbulent flow state has been evaluated $[32,33]$. Formulas to calculate the hydraulic gradient or head loss have been developed based on energy considerations [34,35]. Dimensional analysis methods have been used to predict drops in pressure in the flow of solid-liquid suspensions [36,37]. The hydraulic gradient has been shown to be influenced by the particle size, specific gravity and fluid viscosity [38,39].

The abovementioned engineering practices represent very important experience for similar projects. Some results from the theoretical, experimental and numerical studies are fundamental for understanding the mechanism of the accumulation of plugging by pouring aggregates to control water inrush or salvage underground mines or projects. However, in-depth research is lacking in quantity and universality due to the concealment of underground engineering. Among many controversies, there are some issues commonly raised because they are important for the effective control of groundwater-inrush accidents. One controversy is the selection of the particle size of the aggregates and their pouring order, i.e., whether it is better to pour coarse aggregates first and then introduces the fine ones or vice versa. Another controversy is the order of pouring by boreholes, i.e., whether to pour in the downstream borehole first and then in the upstream borehole or vice versa. Therefore, the first objective of this study was to investigate the effects of the pouring order for the aggregates of different particle sizes on the plugging effects using a visualized experimental setup. The second objective was to investigate the sequence of pouring from different boreholes upstream or downstream of the water inrush point. The results from field investigations were compared with those from experiments. Finally, a suitable pouring order regarding the particle sizes of the aggregates and the borehole locations is discussed and recommended for achieving better plugging. 


\section{Materials and Methods}

\subsection{Materials}

Three groups of aggregate particle sizes in the experiments were selected: $0.25-0.5,0.5-2$ and $2-5 \mathrm{~mm}$. The sand and gravel were screened and washed to remove finer soil and impurities to ensure the clear visualization of the aggregate movement process. Water was added to the funnel to saturate the aggregate to form a water-sand mixture flow. The water-solid mass ratio was maintained between 1:1 and 2:1, and the pouring speed was controlled using a valve, while avoiding hole blocking.

The tunnel replica is made up of an acrylic circular tube unit and an adjustable angle support platform device. The acrylic tube consists of two round tubes with an internal diameter of $200 \mathrm{~mm}$, wall thickness of $10 \mathrm{~mm}$ and length of $2000 \mathrm{~mm}$, in which the middle is connected by a flange and sealing ring. The tunnel model has four boreholes of $25 \mathrm{~mm}$ for pouring and six measurement holes with a diameter of $6 \mathrm{~mm}$ to connect water-pressure sensors. At both ends of the tube, there are high-strength plates with inlet holes and drainage holes. There is a round plate evenly covered with small holes near the inlet and outlet, which can change the water flow state to a uniform pipe flow. A geometric ratio of 20 between the prototype and the model was selected based on the Euler criterion and the gravity similarity criterion. Based on previous research and similarity criteria, the pipeline can be used to simulate a tunnel with a cross-sectional area of $12 \mathrm{~m}^{2}$, length of $80 \mathrm{~m}$ and water-inrush flow of $6440 \mathrm{~m}^{3} / \mathrm{h}[11]$.

\subsection{Experimental Setup}

The efficiency of the plugging achieved by injecting aggregates into a submerged tunnel with flowing water is affected by many factors, including natural and engineering factors, such as the tunnel inclination, water flow rate, distance between the boreholes, aggregate particle size, water-solid mass ratio and injection speed. An experimental setup that could provide a constant water head, and adjustable and recyclable water flow at different flow rates was developed. Figure 1 shows a schematic and photo of the setup. It could control the water-solid mass ratio and pouring speed, as well as collect the data of the flow rate and water pressure in real time. The aggregate accumulation and migration images in the pipeline were captured for process analysis.

\subsection{Scheme of the Experiments}

In order to investigate the influence of the pouring order on the plugging effect of pouring aggregate from multiple boreholes, two groups of tests were devised by considering the order of pouring the aggregates with different particle sizes and locations.

In engineering practice, the orders of pouring finer particles first and coarser particles later and pouring fine particles upstream and coarse ones downstream have been adopted by the majority, but there are still some disagreements [3,4,8,9]. The contrary opinion recommends the order of pouring coarser particles first and finer particles later because the finer particles may fill the voids in between coarser ones and enhance the plugging effect. Therefore, the purpose of Trial No. 1 was to investigate the influence of the pouring orders regarding different times and different particle sizes. The same pouring order was chosen in the field case of this study. The reason for the selection of pouring order in Trial No. 2 is to investigate the influence of different pouring orders in upstream and downstream boreholes. The results from Trial Nos. 1 and 2 could then be compared, which was lacking in the previous investigations. Previous studies focused on the influencing factors for pouring aggregate into a horizontal pipeline through a single borehole but lacked the investigation of pouring via multiple boreholes and in different orders. 


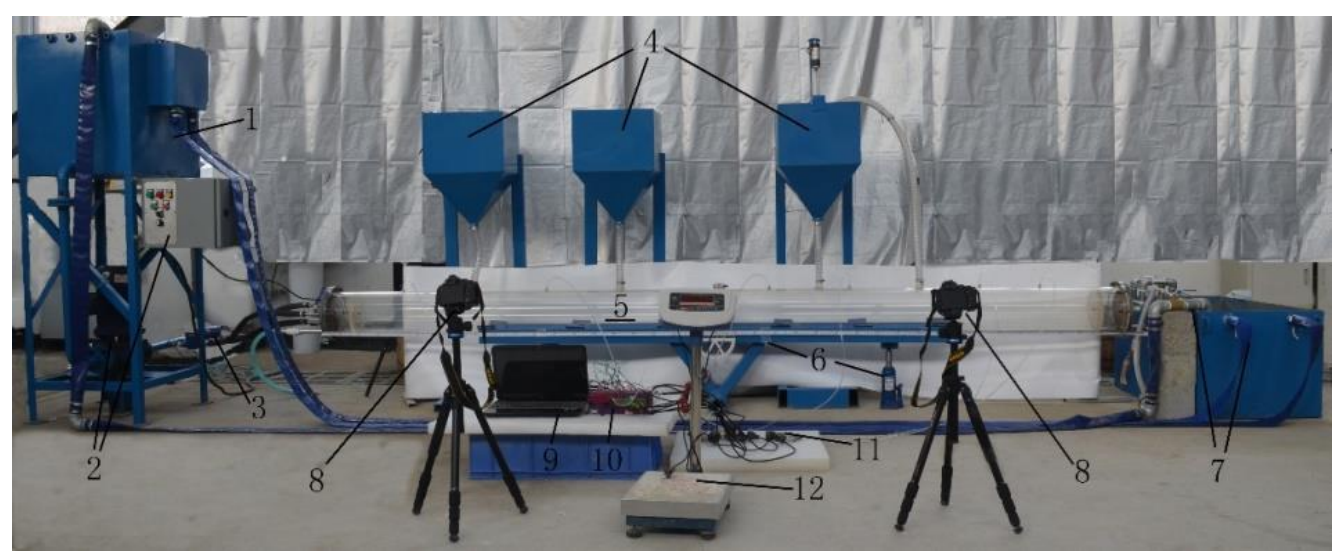

(a) Photo of experimental setup.

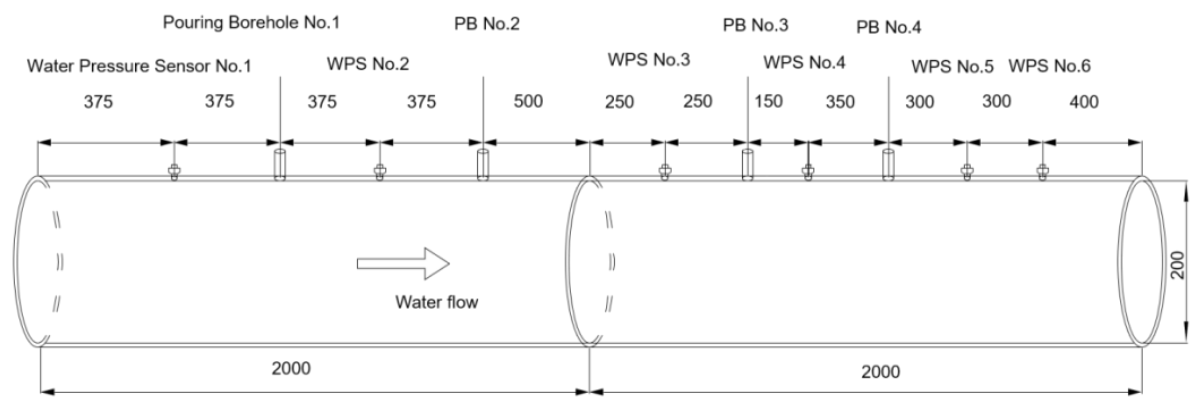

(b) Layout of boreholes and sensors (unit: $\mathrm{mm}$ ).

Figure 1. Experimental setup. 1-water supply with a constant head; 2 -water-flow speed adjustment; 3-water meter; 4-funnel for pouring aggregate; 5-tunnel replica; 6-inclination adjusting; 7-water circulation tank; 8-camera; 9-PC; 10—data recorder; 11—water-pressure sensor; 12—electronic scale.

In the tests, three boreholes were used for pouring and one was used as a vent. Images and data were obtained from cameras, water meters and DataTaker collectors, which were then used to analyze and discuss the influence of different pouring sequences on the efficiency of the plugging. The two groups of trials were as follows:

(1) Trial No. 1 was a trial of pouring fine aggregates first and pouring the coarse ones later, focusing on the pouring order for the different particle sizes at different times. Specifically, Borehole Nos. 1, 2 and 3 were used as pouring boreholes and Borehole No. 4 was used as a vent. The same particle size of aggregate was poured through the three pouring boreholes at the same time. The pouring order was pouring medium sand $(0.25-0.5 \mathrm{~mm})$ first, coarse sand $(0.5-2 \mathrm{~mm})$ second, and gravel sand $(2-5 \mathrm{~mm})$ last, according to the particle sizes used in the field case and similarity criterion. The flow-rate scale was 4.47, calculated from a geometric ratio of 20. The flow speed in the water-inrush tunnel in the Panji coal mine No. 2 is $7 \mathrm{~cm} / \mathrm{s}$. A water flow speed of $1.5 \mathrm{~cm} / \mathrm{s}$ was chosen. In the rescue of flooded mines, blocking is generally implemented in a horizontal tunnel or an uphill tunnel with a small inclination. Therefore, an inclination angle of $0^{\circ}$ for the tunnel in Trial No. 1 was chosen, while $8^{\circ}$ was chosen in Trial No. 2. The process involved pouring medium sand at a rate of $700 \mathrm{~g} / \mathrm{s}$ for $130 \mathrm{~s}$, coarse sand at $270 \mathrm{~g} / \mathrm{s}$ for $165 \mathrm{~s}$ and gravel at $160 \mathrm{~g} / \mathrm{s}$ for $190 \mathrm{~s}$.

(2) Trial No. 2 was a trial of pouring fine particles into the borehole at the upstream location first and then pouring coarse particles in the borehole downstream, focusing on the pouring order for the different particles at different locations relative to the water-inrush point. Borehole Nos. 1, 2 and 3 were the same as those in Trial No. 1, being used as pouring boreholes, and Borehole No. 4 was used as a vent. Different particle sizes of the aggregate were poured into the pouring boreholes at the same time. Medium sand $(0.25-0.5 \mathrm{~mm})$ was poured into Borehole No. 1 ; coarse sand $(0.5-2 \mathrm{~mm})$, into Borehole No. 2; and gravel ( $2-5 \mathrm{~mm}$ ), into Borehole No. 3. A water flow speed of $1.5 \mathrm{~cm} / \mathrm{s}$ was selected, and the inclination angle of the tunnel was $8^{\circ}$. After completing this phase of the experiment, 
the pipe was filled until the portion behind Borehole No. 1 was fully filled. This phase is called the pipeline-filling phase. The pouring rate was $90 \mathrm{~g} / \mathrm{s}$ in Borehole No. 1, $40 \mathrm{~g} / \mathrm{s}$ in Borehole No. 2 and $30 \mathrm{~g} / \mathrm{s}$ in Borehole No. 3, respectively; pouring lasted for $900 \mathrm{~s}$ in all cases.

\subsection{Site Investigation}

On 23 May 2017, at 22:51, an accident of groundwater inrush occurred from a tunnel in the floor of panel 12,123 of the Panji coal mines No.2, Huainan city, Anhui province, China. The flow rate suddenly increased to $3024 \mathrm{~m}^{3} / \mathrm{h}$ at 22:46 on the $25 \mathrm{th}$, and it continued to increase and was greater than the drainage capacity of the coal mine, causing the mine to be flooded. It was estimated that the water flow rate was $14,000 \mathrm{~m}^{3} / \mathrm{h}$ immediately after the flooding.

During the water inrush, the water levels in the observation boreholes of the Ordovician limestone aquifer changed greatly. For instance, by 27 May, the water level in a borehole $1170 \mathrm{~m}$ away from the inrush area decreased by $145.11 \mathrm{~m}$; in another borehole $1400 \mathrm{~m}$ away, it decreased by $133.52 \mathrm{~m}$. Since there was no large fault structure explored in the water-inrush area, the pathway of the water should have been a smaller hidden Karst column.

The option of pouring aggregate and grouting reinforcement treatment was chosen from the different options for salvaging the mine. During the treatment period, the water source was explored and sealed. The water-blocking project was completed at the end of August. A water-blocking section in the roadway was constructed, and the water source was blocked. In this study, the observations and investigations during the pouring process will be analyzed and compared to experimental results to obtain relevant understanding.

\section{Results}

The plugging of water by pouring aggregate means that an accumulated aggregate mass with a certain length and water resistance capacity has formed, when enough aggregate has been poured. At this point, the flow rate decreases and the pipe flow in the tunnel becomes a seepage flow. The seepage flow herein refers to the water flow that passes through the porous medium made of aggregates. There is an obvious water-pressure difference between the front and back of the accumulated mass. This situation indicates that the plugging of water with pouring aggregate is successful. The efficiency of plugging and the main effects of pouring aggregate in a flowing tunnel have been discussed in the literature [11].

\subsection{Effect of the Pouring Order with Different Particles at Different Times}

Figure 2 shows the pouring and accumulation process in Trial No. 1, in which the medium sand was poured first from Borehole Nos. 1, 2 and 3 at the same time. Three accumulation masses were preliminarily formed, which adopt a cone shape in the tunnel replica (Figure 2a,b). Then, the pouring material was replaced with coarse sand. The deposition of the coarse sand connected the cone-shaped accumulated masses, until an aggregate accumulated mass with a steady water-flow channel formed (Figure 2c,d). During this period, the residual water channel continuously decreased. Finally, the gravel particles were poured instead of the coarse sand. The plugging became a capping stage. However, in this stage, most of the poured gravel was carried directly downstream by water, sliding over the surface of the accumulated mass. The gravel hardly accumulated in the tunnel and completely blocked the high-speed water flow (Figure 2e). 


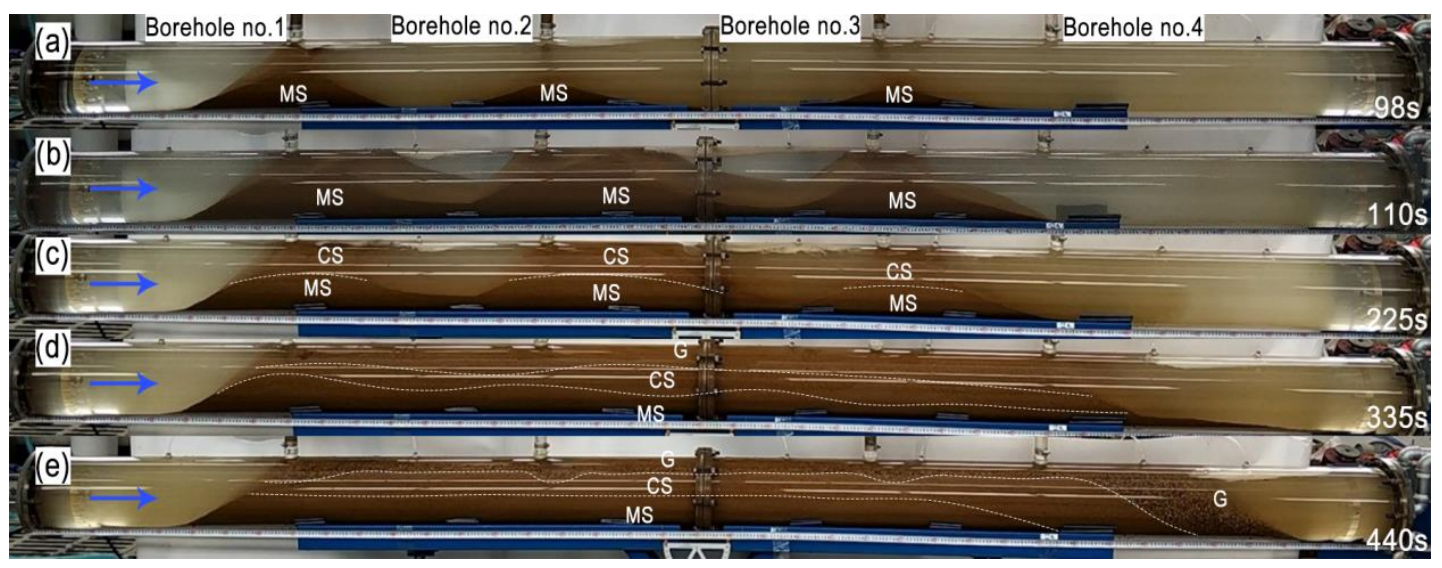

Figure 2. Aggregate deposits in Trial No. 1, with the order of pouring fine particles first and coarse particles later. MS - medium sand; CS-coarse; G-gravel. (a) at $98 \mathrm{~s}$, (b) at $110 \mathrm{~s},(\mathbf{c})$ at $225 \mathrm{~s}$, (d) at $335 \mathrm{~s},(\mathbf{e})$ at $440 \mathrm{~s}$.

Figure 3 shows an explanationary diagram of the critical conditions for the movement of particles into the sedimented and accumulated mass in a horizontal tunnel and inclined tunnel.

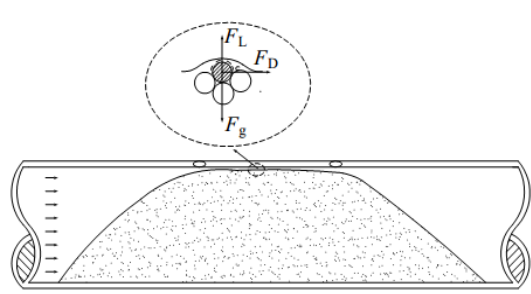

(a)

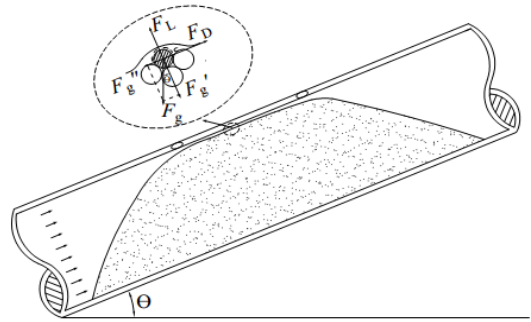

(b)

Figure 3. Force on particle from movement to sedimentation in a (a) horizontal tunnel and (b) inclined tunnel.

Effective gravity,

$$
F_{\mathrm{g}}=\frac{1}{6} \pi d_{\mathrm{s}}^{3}\left(\rho_{\mathrm{s}}-\rho_{\mathrm{w}}\right) g
$$

Drag force in the direction of water flow,

$$
F_{\mathrm{D}}=C_{\mathrm{D}} \frac{1}{8} \pi d_{\mathrm{s}}{ }^{2} \rho_{\mathrm{w}} u_{\mathrm{w}}{ }^{2}
$$

Lift force,

$$
F_{\mathrm{L}}=C_{\mathrm{L}} \frac{1}{8} \pi d_{\mathrm{s}}^{2} \rho_{\mathrm{w}} u_{\mathrm{w}}^{2}
$$

Critical condition for particle settelement and accumulation:

$$
\begin{gathered}
F_{\mathrm{D}} \leq \mu\left(F_{\mathrm{g}}-F_{\mathrm{L}}\right) \\
u_{\mathrm{w}}{ }^{2} \leq \frac{4}{3} \frac{g d_{\mathrm{s}}\left(\rho_{\mathrm{s}}-\rho_{\mathrm{w}}\right)}{\rho_{\mathrm{w}}} \frac{\mu}{C_{\mathrm{D}}+\mu C_{\mathrm{L}}}
\end{gathered}
$$

For an inclined tunnel:

$$
\begin{gathered}
F_{\mathrm{D}} \leq F_{\mathrm{g}}{ }^{\prime \prime}+\mu\left(F_{\mathrm{g}}{ }^{\prime}-F_{\mathrm{L}}\right) \\
u_{\mathrm{w}}{ }^{2} \leq \frac{4}{3} \frac{g d_{\mathrm{s}}\left(\rho_{\mathrm{s}}-\rho_{\mathrm{w}}\right)}{\rho_{\mathrm{w}}} \frac{\sin \theta+\mu \cos \theta}{C_{\mathrm{D}}+\mu C_{\mathrm{L}}}
\end{gathered}
$$


where, $C_{\mathrm{D}}$ is the drag force coefficient, $C_{\mathrm{L}}$ is the lift force coefficient, $u_{\mathrm{w}}$ is the average flow speed in the residual channel, $\mu$ is the sliding frictional coefficient, $\theta$ is the tunnel inclination, $d_{\mathrm{s}}$ is the particle size, $\rho_{\mathrm{s}}$ is the density of the particles, $\rho_{\mathrm{w}}$ is the density of water, and $g$ is the accelaration of gravity.

The wall of the pipe is smooth, and the friction resistance $\mu$ between the aggregate and the bed-sand surface is not sufficient to retain the gravel. The flow rate in the residual channel is large enough to move the particle. This is why the aggregates are difficult to accumulate for cutting off the flow of water.

Figure 4 shows the changes in fluid pressure in the tunnel during pouring. The effects of the build-up of the aggregate mass on the water flow can be reflected by the changes in water pressures. The curve in the figure can be divided into three stages: pouring medium sand, coarse sand and gravel. In the figure, $t_{0}$ is the moment of starting the pouring of medium sand, $t_{1}$ is the time for which the coarse sand is poured, $t_{2}$ is the time for which the gravel is poured, and $t_{\text {final }}$ is the moment at which the build-up of accumulated mass is stabilized. With the injection of aggregates with different particle sizes, the water pressure in the tunnel showed an increasing trend overall. At $t_{0}$, as the sand was poured in, the aggregates accumulated under the action of gravity, and the overall water pressures rose. At the $t_{1}$ moment, the pouring of coarse sand connected the accumulated sand masses until the height of the mass was no longer increased. The water pressures at Water Pressure Sensors No. 1 and 2 increased and began to exceed those at Water Pressure Sensors 3, 4, 5 and 6, while the pressure fluctuations were obvious. At the $t_{2}$ moment, the tunnel was filled with gravel for capping. Although the efficiency of water plugging was unsatisfactory, the pressure at Water Pressure Sensors No. 1 and 2 began to rise and maintain large fluctuations, while the pressure at Water Pressure Sensors 3 , 4, 5 and 6 exhibited a downward trend with large fluctuation. This clearly indicates the obstructing effect of the accumulated aggregate mass on water flow. In order to achieve more efficient plugging, pouring aggregates with larger particle sizes or pouring faster is required at this point. At the $t_{\text {final }}$ moment, the build-up of accumulated aggregate mass in the tunnel is stable when the water channel does not change again.

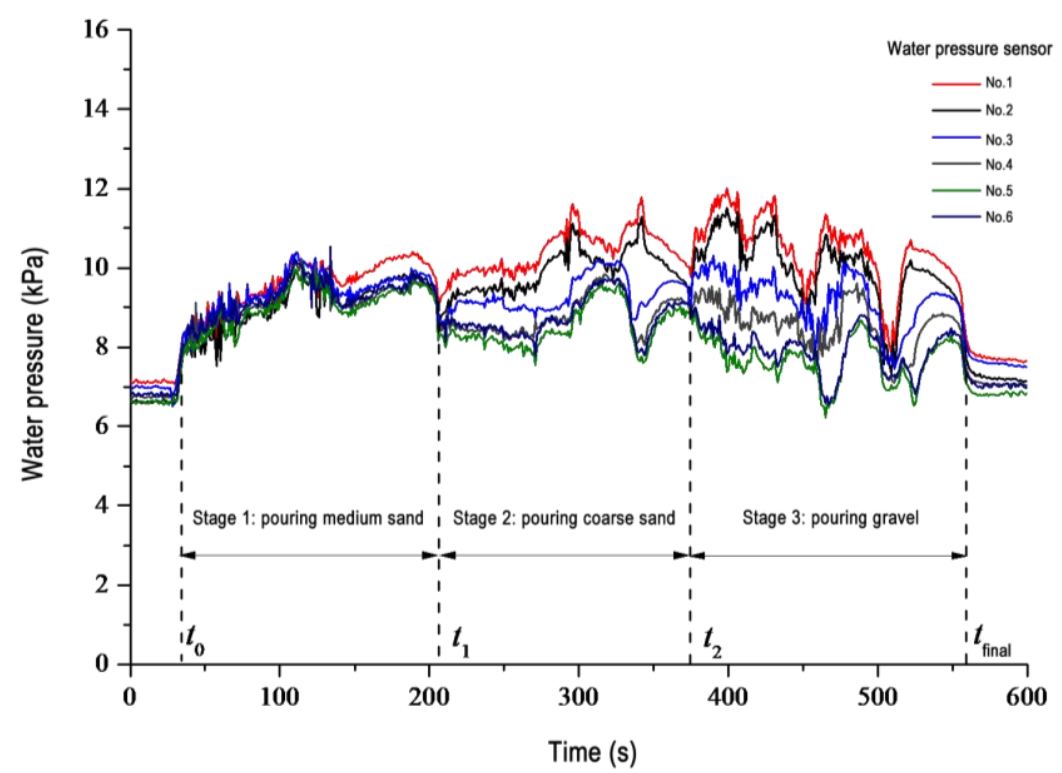

Figure 4. The water pressure in the tunnel with the order of pouring fine particles first and coarse particles later.

\subsection{Effect of the Pouring Order for Different Particles at Different Locations}

Figure 5 shows the pouring and accumulation process of Trial No. 2. The plugging effect was investigated in an inclined tunnel, pouring aggregate of different particle sizes in the order of pouring fine gains upstream and coarse grains downstream. The effect of inclination can be analyzed by 
comparing Equations (5) and (7). The effect of inclination on the critical condition of aggregate deposition is small and can be neglected when $\theta<10^{\circ}$. The pouring process was implemented in two stages. In the first stage, medium sand was poured from Borehole No. 1; coarse sand, from No. 2; and gravel, from No. 3. During the pouring process, the gravel deposited the fastest, with coarse sand ranking second, and the medium sand was the slowest. They all deposited beneath the location of the pouring borehole with a cone shape. However, as the upstream medium sand reached a certain height, the flow rate of the water accelerated. The carrying capacity of the water flow was strengthened, and the medium and coarse sands were mainly moved by suspension and bed load. When the sand flow moved to the top of the gravel stack, the accumulated gravel mass was directly washed away. Gravel was also carried downstream; the capping and plugging were unsuccessful.

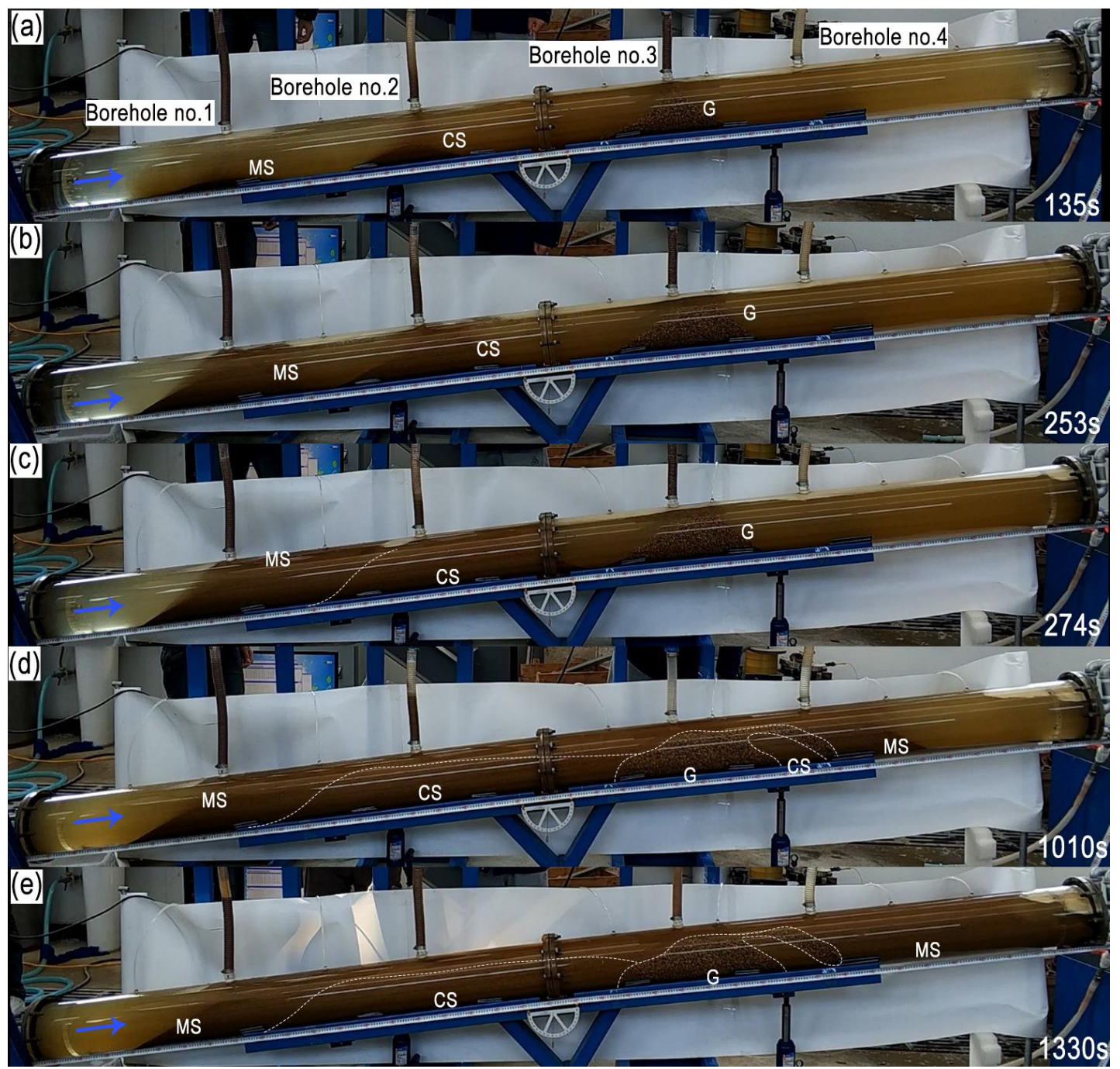

Figure 5. Aggregate deposits in Trial No. 2, with the order of pouring fine particles upstream and coarse particles downstream. (a) at $135 \mathrm{~s},(\mathbf{b})$ at $253 \mathrm{~s},(\mathbf{c})$ at $274 \mathrm{~s},(\mathbf{d})$ at $1010 \mathrm{~s},(\mathbf{e})$ at $1330 \mathrm{~s}$.

In the second stage, the poured aggregate continued to fill the whole tunnel. The accumulated aggregate mass finally extended to the outlet, but the sand flowed out of the outlet. This implies that the sand would flow into the other section of the tunnel if it was long enough, indicating unsuccessful plugging. Otherwise, if the tunnel was shorter, the sand would have been blocked by the end of the tunnel. On the one hand, the reason is that the drag force for moving the fine particles was small due to the smooth wall of the pipe. The finer grains are easy to set in motion and break away from the accumulated mass of coarser grains downstream. Equations (4) and (6) can be used for a 
theoretical explanation. The CFD (Computational Fluid Dynamics) models reviewed and developed by Messa and Matoušek [18] could be used to evaluate the drag force acting on the particles in a slurry in a further study. In the pipeline transportation of slurry, fine grains of a certain concentration can reduce the resistance. The coarse particles are difficult to deposit and accumulate on the sand bed; they move forward along the sand bed. In addition, the pumping pressure in the test was constant, to simulate the water flow. In practical engineering, there is a decline in water pressure, which is beneficial for the deposition of aggregates.

Figure 6 shows the changes in fluid pressure during pouring, with the order of pouring fine particles upstream and coarse ones downstream. In the figure, $t_{0}$ is the moment of starting to pour the particles, $t_{1}$ is the time of the beginning of the second stages, and $t_{\text {final }}$ is the stop time. In the $t_{0}$ moment, after a sudden increase in fluid pressure, the pressure continued to decline. At time $t_{1}$, the aggregates continued to be poured, and the newly poured aggregates accumulated downstream. The resistance from the early deposited accumulation mass causes large fluctuations in water pressure. The water flow rate is high enough to move the aggregates, so they are difficult to accumulate. The plugging effect is poor. It can be found that the pressure difference at the aggregate capping moment in Trial No. 1 is relatively small compared to that in Trial No. 2, implying a different plugging effect.

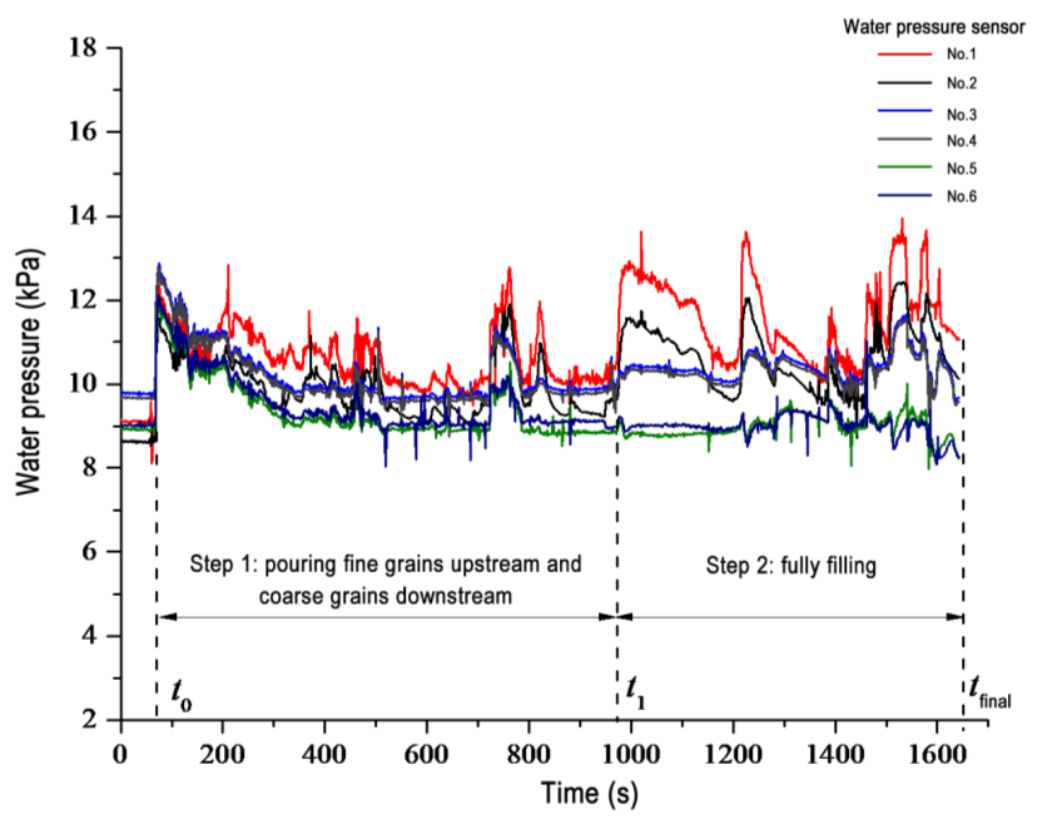

Figure 6. The water pressure in the tunnel, with the order of pouring fine particles upstream and coarse particles downstream.

\subsection{Performance of Pouring Aggregate in the Field}

Figure 7a shows a schematic for pouring aggregates and then grouting in a coal mine tunnel for the salvage of an inundated mine. Figure $7 \mathrm{~b}$ shows the relationship of the layout of pouring boreholes and the tunnel of water inrush in the Panji coal mine No. 2. Due to the presence of multiple mining areas above the water-inrush point, the ground was covered by a large-scale subsidence basin filled with water. It was very difficult to cross the multi-layer mined-out area using the straight boreholes directly from the ground. Therefore, a ground directional borehole with several branches from the conditional ground position was selected for drilling into the tunnel near the water-inrush point. The pouring of aggregate and grouting was then implemented to cut off the water flow. Figure 7c shows the poring funnel on ground. 


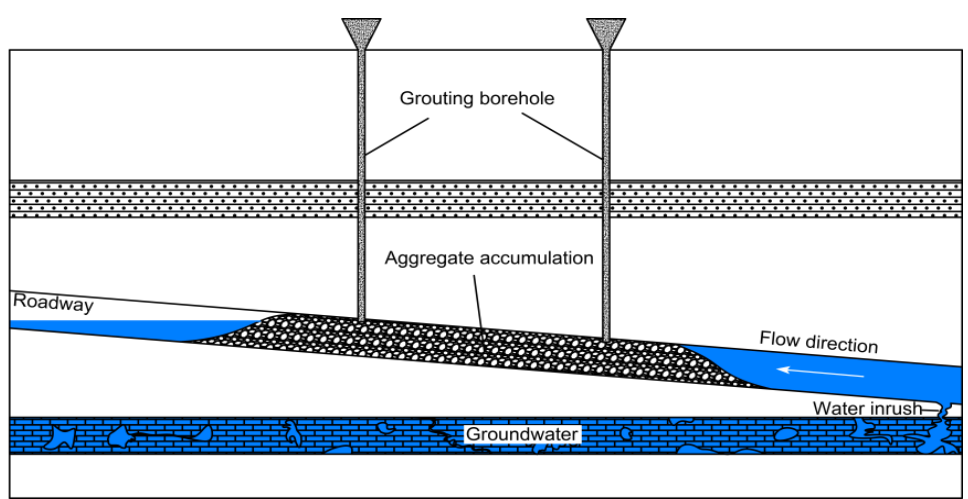

(a)

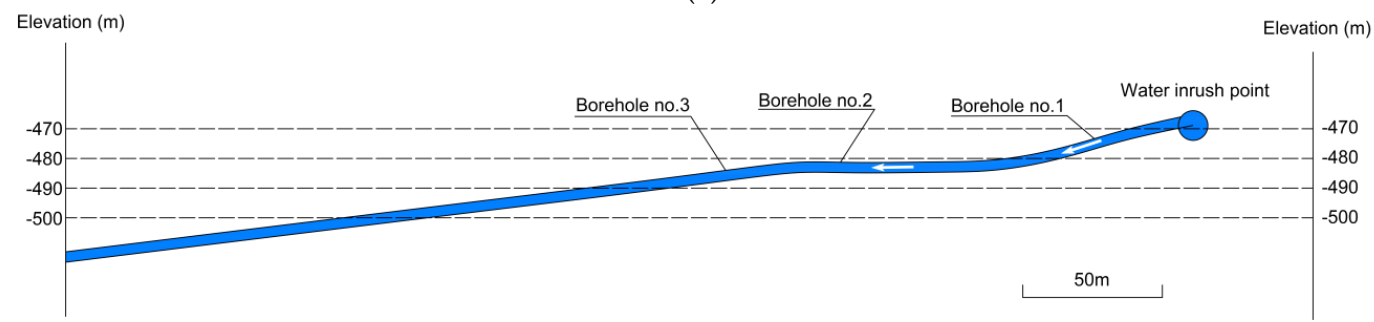

(b)

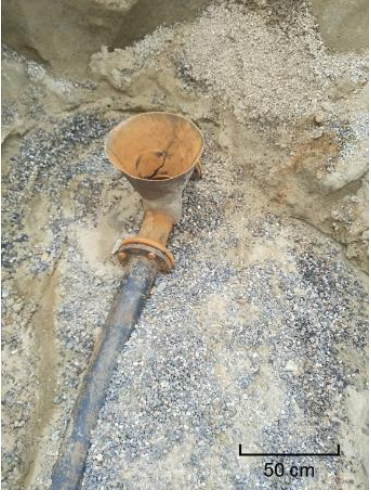

(c)

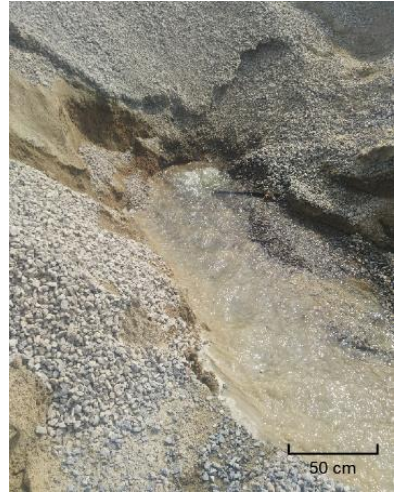

(d)

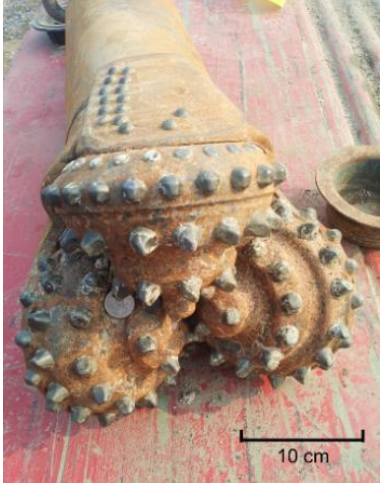

(e)

Figure 7. (a) Schematic diagram of pouring aggregates to plug a roadway; (b) layout of pouring boreholes; (c) pouring funnel; (d) water flow out of borehole due to blockage; (e) a roller drill.

Aggregate, cement and fly ash were chosen as the materials for this pouring and grouting. The project consumed $2429 \mathrm{~m}^{3}$ of sand and gravel with a particle size of $0.7-3.0 \mathrm{~mm}, 4867 \mathrm{~m}^{3}$ of gravel with a particle size of $5-10 \mathrm{~mm}, 553 \mathrm{~m}^{3}$ of gravel with a particle size of $10-20 \mathrm{~mm}$ and $1104 \mathrm{~m}^{3}$ with a size of $30-50 \mathrm{~mm}$. The amount of cement used for grouting was 15,515.88 tonnes. A final pumping pressure of $6 \mathrm{MPa}$ was chosen for the grouting.

Aggregates of various particle sizes should be prepared in advance because the pouring of aggregate must be continuous, and the amount of aggregate required is relatively large. The aggregate enters the jet through the funnel and fills the tunnel through the borehole, which often becomes blocked during pouring. Figure $7 \mathrm{~d}$ shows the flow of water out of the borehole due to blockage. If the ground aggregate-injection pipe is blocked, the pipe can be removed to poke it out; if the borehole is blocked, it can only be unblocked by drilling and sweeping it. Figure 7e is a roller drill that is needed to dislodge the large gravel, and at the same time, a larger concentration of drilling fluid is required. It is necessary to set up a vent borehole during the pouring process. Because of the continuous pouring of aggregate, the air pressure in the tunnel and the borehole is increased by the injection. The air is exhausted through the injection port; a small fountain sometimes occurs. 
The order of aggregate pouring adopted in this project was similar to that in Trial No. 1. Through the pouring of fine materials in the early stage, the cross-sectional area of the roadway was reduced and the water flow speed was increased. The aggregate particle size was gradually increased in the later stage to form an effective water-barrier section in the tunnel. After the successful plugging of the roadway by pouring aggregates, cement grouting was used for filling and pressure-increasing reinforcement. Finally, a water-plug section with a length of $106 \mathrm{~m}$ was formed and the water plugging was completed, with a sealing efficiency of $100 \%$.

\section{Discussion}

In this experiment, two pouring sequences were selected to study their effects on water plugging with aggregates of different particle sizes. The mixed pouring of aggregates of different particle sizes, whether fine aggregates will fill the gaps of coarse aggregates, and whether different plugging effects will occur have been meaningfully discussed.

Due to the different conditions of coal mines, plans for the treatment of water inrushes with shutoff projects in the submerged mine roadways have their own characteristics. There are roughly three options for the treatment of a major water inrush. The first is to plug the concentrated water passage in the water-inrush area first and then block the water-inrush source and any nearby main water-inrush pathways with grouting. The second is to block the source as the mainstay and block the flow as a supplement. The purpose is to establish a water plug below the water-inrush pathway such as a collapsed column. The third is to cut off the flow of water first and then establish a water stop plug below the Karst collapsed column [4].

For submerged large-sectional-area roadways of mines, the construction of the resistance section for the concentrated water passage requires the filling of a very large space. For a very large water inrush, the length of the resistance section is generally several hundred meters. The construction of the resistance section-flowing water grouting and water shutoff work-generally involves aggregate pouring and grouting reinforcement.

The process of aggregate accumulation and plugging is considered to be divided into three stages in the literature: the bottom-laying stage, filling stage and plugging stage $[3,40]$. The water velocity is low in the bottom-laying stage; finer aggregates are selected to extend as far as possible along with the water, settling on the bottom of the tunnel. In the filling stage, at a certain water pressure and flow rate, the cross-section of the channel decreases continuously with aggregate pouring, and the water flow speed increases accordingly. The particle size is increased to quickly fill the remaining channel of the tunnel. In the plugging stage, approaching the capping, the battle between aggregate capping and water flushing always exists. When capping with multiple boreholes connected to each other with a certain length, the filling section is able to resist the water pressure. The minimum extension length is tried in order to achieve the best water-blocking efficiency. The key to capping is to follow the pouring plan and increase the pouring intensity as much as possible, choosing the same or larger particle size of aggregates. When there is a significant drop in the water level in the front and back of the resistance section, the critical moment for filling and capping is approaching. After successful capping, the aggregate pouring speed and particle size are adjusted continuously to further fill the pores in the accumulated mass, reduce the seepage speed and create favorable conditions for grouting.

The key aspects of the construction technology for aggregate pouring include preventing the borehole from being blocked, avoiding the spraying of the borehole, drilling the borehole with mud protection, and selecting an appropriate aggregate particle size and water-solid mass ratio.

According to engineering practice, the key to pouring aggregate is a reasonable combination of aggregate particle sizes. Generally, the aggregate particle size and the order of pouring depend on the flow rate of the moving water, but they are also related to the geological conditions and environment of the water-inrush roadway, such as whether the roadway has caving or collapsed rocks. This is a favorable location for water blocking. Priority is given to the injection of fine-grained aggregates for covering the floor and extension accumulation. Finally, the coarse and fine aggregates are combined 
with pouring, and the sequence is that of pouring fine aggregates first and coarse ones later, similar to that employed in this experimental study. Another example is the treatment of water inrush in the Sangshuping coal mine in Shaanxi province. In that project, coarse and fine aggregates were chosen, and the flow was successfully blocked by the pouring of fine aggregate.

There are still many problems worthy of future in-depth study. There are many differences between the controlled, laboratory tests and the more complex field case. For example, the speed of pouring the aggregate in this experiment was different from the actual one. Generally, the actual tunnel cross section is not circular. The sedimentation and migration of particles in different cross-sectional shapes will differ. In addition, the water pressure in this test was too low. Certain properties of the high-pressure water flow influence aggregate accumulation, including the flow regime, density and permeability. Another limitation is that the model wall was smooth and roughness was not considered. If we assume that $\mu=1$, the error between Equations (5) and (7) is $13 \%$ for an inclination of $8^{\circ}$. Therefore, the two results can be compared with each other in these two specific cases. However, increasing the inclination angle will significantly affect the results. These aspects might make the laboratory results not directly applicable to the field case and make their practical applicability uncertain. Therefore, a cross-sectional shape similar to that of an actual tunnel should be chosen when modeling the pouring of aggregate into flooded tunnels.

Further research should be conducted to investigate the effect of high-pressure water flow on the properties of aggregate accumulation using the newly developed equipment [12]. The effect of different roughness on the blocking effect of the accumulated aggregate mass, under the premise of ensuring visualization, should also be followed up. More cases including different orders of pouring will be investigated experimentally or numerically.

\section{Conclusions}

A visualized test platform for the process of aggregate pouring in a tunnel was used to investigate the effect of the pouring order on the efficiency of plugging through multiple boreholes under a flowing-water condition. A case study was compared with the experimental results. The main conclusions are as follows:

(1) In the experiments of water plugging with different orders of pouring aggregates, it was found that the efficiency of plugging with the order of pouring fine aggregate first and coarse aggregate later was better than that with the order of pouring fine aggregate upstream and coarse aggregate downstream. When the poured aggregate is about to be capped, increasing the intensity of pouring with the same or a larger particle size is more conducive to capping.

(2) The build-up of aggregate mass in a tunnel with flowing water can be reflected in the change in water pressure. It could be divided into three stages-pouring medium sand, coarse sand and gravel-in Trial No. 1, with the pouring of fine aggregate first and coarse aggregate later. The water pressure difference at the aggregate-capping moment in Trial No. 1 was relatively small compared to that in Trial No. 2, with the order of pouring fine particles upstream and coarse ones downstream. This implies that the efficiency of plugging with the order of pouring fine aggregate first and coarse aggregate later (the fine-coarse order) in different boreholes was better than that with the order of pouring fine aggregate upstream and coarse aggregate downstream (the fine-upstream-coarse-downstream order).

(3) A successful case of flooded-mine salvage using ground directional boreholes to pour aggregate and grouting to cut off the water flow shows that pouring fine materials in the early stage reduced the cross-sectional area; the aggregate particle size was gradually increased in the later stage to form an effective water-barrier section in the tunnel. Blockages in the borehole can be dealt with by drilling and sweeping the hole. It is necessary to set up a vent borehole during the pouring process to release compressional pressures. The poured aggregate provided a base for cement grouting to form a water-plug section with a length of $106 \mathrm{~m}$, resulting in a sealing efficiency of $100 \%$. 
Author Contributions: X.J. and W.S. conceived the main idea of the paper and designed the test scheme; S.H. conducted the tests; S.H. and W.S. analyzed the results; X.J., W.S. and S.H. wrote the paper; Z.S. and J.W. performed the field project and provided the field data. All authors proofread and modified the manuscript. All authors have read and agreed to the published version of the manuscript.

Funding: This research was funded by the Natural Science Foundation of China under Grant No. 4177283.

Acknowledgments: The authors are thankful for the assistance from Ph.D. student Yankun Liang and graduate students Shouliang Zhao, Guosheng Zheng, Shichong Yuan, Haiqing Liu and Cong Xie of the CUMT for the help during testing. The editorial help from graduate students Jiaxing Chen and Jinchuan Zhang of the CUMT is also acknowledged.

Conflicts of Interest: The authors declare no conflict of interest.

\section{References}

1. Jiang, Q. Comprehensive construction technology of water-blocking bulkhead in the with concentrated water flow. Coal Mine Saf. 2009, 40, 37-39.

2. Li, C.H. Plugging technology for super giant water inrush laneway in mine. J. Xi'an Univ. Sci. Technol. 2010, 30, 305-308.

3. Wang, W. Study on Techniques of Roadway-Blocking \& Flow-Cutting off under Hydrodynamic Conditions and Capability Evaluation of Water-Blocking Segment; Coal Science Research Institute: Beijing, China, 2012.

4. Guo, Q. Technology for Rapid Treatment of Major Coal Mine Water Hazards: Practice and Knowledge of Grouting to Block Water; Coal Industry Press: Beijing, China, 2005.

5. Ji, Z. Grouting and water blocking technology under complex conditions of water inrush in coal mine. Explor. Eng. 2014, 41, 61-65.

6. Shao, H.; Wang, W. Double liquid grouting method for rapid construction of water blocking bulkhead to block water inrush roadway. Coal Mine Saf. 2011, 42, 40-43.

7. Wang, W.; Hu, B. A new technology of rapid sealing roadway in the Luotuoshan Coal Mine. Procedia Earth Planet. Sci. 2011, 3, 429-434. [CrossRef]

8. Wang, S.; Wei, Z.; Lei, F.; Xue, G.; Tong, X.; Li, Y. Study on coalmine hidden extraordinary water bursting channel instant sealing technology. China Coal Geol. 2013, 25, 31-35.

9. Zheng, S. Application of ground directional borehole to control prevention karst collapsed column water inrush in coalmines. Coal Sci. Technol. 2018, 46, 229-233.

10. Zhu, M. Key technology and equipment of borehole-controlled grouting for highly effective plugging large channel of water inrush. Coalf. Geol. Explor. 2015, 43, 55-58.

11. Zhang, G.; Hui, S.; Sui, W.; Li, W. Experimental investigation on pouring aggregate to plug horizontal tunnel with flow water. Water 2020, 12, 1763. [CrossRef]

12. Dong, S.; Yang, Z.; Zhu, M.; Zhang, W.; Shi, L.; Mou, L. Development of large-scale simulation experiment system of dynamic water rapid sealing in flowing water roadway. J. China Coal Soc. 2020. first published on line at 2020-07-11. 1-11. [CrossRef]

13. Kaushal, D.; Thinglas, T.; Tomita, Y.; Kuchii, S.; Tsukamoto, H. CFD modeling for pipeline flow of fine particles at high concentration. Int. J. Multiph. Flow 2012, 43, 85-100. [CrossRef]

14. Capecelatro, J.; Desjardins, O. Eulerian-Lagrangian modeling of turbulent liquid-solid slurries in horizontal pipes. Int. J. Multiph. Flow 2013, 55, 64-79. [CrossRef]

15. Arolla, S.K.; Desjardins, O. Transport modeling of sedimenting particles in a turbulent pipe flow using Euler-Lagrange large eddy simulation. Int. J. Multiph. Flow 2015, 75, 1-11. [CrossRef]

16. Uzi, A.; Levy, A. Flow characteristics of coarse particles in horizontal hydraulic conveying. Powder Technol. 2018, 326, 302-321. [CrossRef]

17. Messa, G.V.; Malin, M.R.; Malavasi, S. Numerical prediction of fully-suspended slurry flow in horizontal pipes. Powder Technol. 2014, 256, 61-70. [CrossRef]

18. Messa, G.V.; Matoušek, V. Analysis and discussion of two fluid modelling of pipe flow of fully suspended slurry. Powder Technol. 2020, 360, 747-768. [CrossRef]

19. El Mohtar, C.S.; Yoon, J.; Sangroya, R.; Jaffal, H. Transferring innovative research into practical wisdom: The case of permeation grouting. Innov. Infrastruct. Solut. 2017, 2, 37. [CrossRef] 
20. Jaffal, H.A.; Ward, K.K.; Mohtar, C.E. Cement Suspension Flow through Heterogeneous Porous Media. In Geo-Congress 2020: Foundations, Soil Improvement, and Erosion; American Society of Civil Engineers: Reston, VA, USA, 2020. [CrossRef]

21. Civan, F. Chapter 8-Particulate Processes in Porous Media. Reservoir Formation Damage; Elsevier Inc.: Amsterdam, The Netherlands, 2015. [CrossRef]

22. El Mohtar, C.S.; ElKhattab, M.; Sangroya, R. Post-grouting stability of bentonite suspensions within sand pores. Geotech. Spec. Publ. 2015, 256, 2296-2305. [CrossRef]

23. Chequer, L.; Bedrikovetsky, P.; Badalyan, A.; Gitis, V. Water level and mobilisation of colloids in porous media. Adv. Water Resour. 2020, 143, 103670. [CrossRef]

24. Lenchenkov, N.; Glasbergen, G.; Van, K.C. Flow of a cross-linking polymer in porous media. Transp. Porous Media 2018, 124, 943-963. [CrossRef]

25. Civan, F.; Vafai, K. A review of approaches for describing gas transfer through extremely tight porous media. In AIP Conference Proceedings; American Institute of Physics: College Park, MD, USA, 2010; Volume 1254, pp. 53-58. [CrossRef]

26. Durand, R. The Hydraulic Transportation of Coal and Other Materials in Pipe; Colloquium of National Coal Board: London, UK, 1952.

27. Wasp, E.J.; Kenny, J.P.; Gandhi, R.L. Solid-Liquid Flow: Slurry Pipeline Transportation; Trans Tech Publications: Clausthal-Zellerfeld, Germany, 1977.

28. Fei, X.J. Hydraulics of Slurry and Granular Material Transport; Tsinghua University Press: Beijing, China, 1994.

29. Soepyan, F.B.; Cremaschi, S.; Sarica, C.; Subramani, H.J.; Kouba, G.E. Solids transport models comparison and fine-tuning for horizontal, low concentration flow in single-phase carrier fluid. AIChE J. 2013, 60, 76-122. [CrossRef]

30. Zouaoui, S.; Djebouri, H.; Mohammedi, K.; Khelladi, S.; Aider, A.A. Experimental study on the effects of big particles physical characteristics on the hydraulic transport inside a horizontal pipe. Chin. J. Chem. Eng. 2016, 24, 317-322. [CrossRef]

31. Miedema, S.A. The heterogeneous to homogeneous transition for slurry flow in pipes. Ocean Eng. 2016, 123, 422-431. [CrossRef]

32. Ni, J.R.; Wang, G.Q.; Zhang, H.W. Basic Theory of Two-Phase Flow and Its Latest Application; Beijing Science Press: Beijing, China, 1989.

33. Vlasák, P.; Kysela, B.; Chara, Z. Flow structure of coarse-grained slurry in a horizontal pipe. J. Hydrol. Hydromech. 2012, 60, 115-124. [CrossRef]

34. Newitt, D.M.; Richardson, J.F.; Abbott, M.; Turtle, R.B. Hydraulic conveying of solids in horizontal pipes. Trans. Inst. Chem. Eng. 1955, 33, 93-110.

35. Miedema, S.A. A head loss model for slurry transport in the heterogeneous regime. Ocean Eng. 2015, 106, 360-370. [CrossRef]

36. Turian, R.M.; Yuan, T.-F. Flow of slurries in pipelines. AIChE J. 1977, 23, 232-243. [CrossRef]

37. Turian, R.M.; Yuan, T.-F.; Mauri, G. Pressure drop correlation for pipeline flow of solid-liquid suspensions. AIChE J. 1971, 17, 809-817. [CrossRef]

38. Duckworth, R.A.; Argyros, G. Influence of density ratio on the pressure gradient in pipes conveying suspensions of solids in liquids. In Proceedings of the Hydrotransport 2: The 2nd International Conference on the Hydraulic Transport of Solids in Pipes, Coventry, UK, 20-22 September 1972; BHRA Fluid Engineering: New York, NY, USA, 1972.

39. Allahvirdizadeh, P.; Kuru, E.; Parlaktuna, M. Experimental investigation of solids transport in horizontal concentric annuli using water and drag reducing polymer-based fluids. J. Nat. Gas Sci. Eng. 2016, 35, 1070-1078. [CrossRef]

40. Mou, L.; Dong, S.; Zhou, W.; Wang, W.; Li, A.; Shi, Z. Data Analysis and Key Parameters of Typical Water Hazard Control Engineering in Coal Mines of China. Mine Water Environ. 2020, 39, 331-344. [CrossRef]

(C) 2020 by the authors. Licensee MDPI, Basel, Switzerland. This article is an open access article distributed under the terms and conditions of the Creative Commons Attribution (CC BY) license (http://creativecommons.org/licenses/by/4.0/). 